EPJ Web of Conferences 66, 03086 (2014)

DOI: 10.1051/epjconf/20146603086

(C) Owned by the authors, published by EDP Sciences, 2014

\title{
Scattering of light halo nuclei on heavy target at energies around the Coulomb barrier
}

O. Tengblad ${ }^{1, a}$, M.J.G. Borge ${ }^{1}$, M. Cubero ${ }^{1}$, E. Nacher ${ }^{1}$, V. Pesudo ${ }^{1}$, A. Perea ${ }^{1}$, J. GomezCamacho $^{2,3}$, A. M. Moro², J.P. Fernandez-Garcia ${ }^{2,3}$, M.A.G. Alvarez ${ }^{2,3}$, M. Rodriguez-Gallardo², J.A. Lay ${ }^{2}$, I. Martel ${ }^{4}$, L. Acosta ${ }^{4}$, A. M. Sanchez-Benitez ${ }^{4}$, G. Marquinez-Duran ${ }^{4}$, P. Walden ${ }^{5}$, and the E1104 and S1202 collaborations at TRIUMF

${ }^{1}$ Instituto de Estructura de la Materia, CSIC, Madrid, Spain

${ }^{2}$ Centro Nacional de Aceleradores (U. Sevilla, J. Andalucia, CSIC), Sevilla, Spain

${ }^{3}$ Departamento de FAMN, Universidad de Sevilla, Spain.

${ }^{4}$ Departamento de Fisica Aplicada, Universidad de Huelva, Spain

${ }^{5}$ TRIUMF, V6T2A3 Vancouver, British Columbia, Canada

\begin{abstract}
.
We report here on experiments performed at TRIUMF to study the scattering of the light halo nuclei ${ }^{11} \mathrm{Li}$ on lead at energies below and around the Coulomb barrier. The the elastic and break-up differential cross section are interpreted in the framework of ContinuumDiscretized Coupled-Channel calculations. The departure from Rutherford scattering at energies below the barrier is well beyond the behavior of normal nuclei.
\end{abstract}

\section{Introduction}

Nuclear structure properties have often been deduced from nuclear reaction studies. With the advent of the first accelerated radioactive beams, new nuclear structures were discovered, such as the existence of a halo in some very loosely bound nuclei. The halo structure is a threshold phenomenon due to the low binding energy of the last nucleons. Halo nuclei have several features in common, such as a rather compact core, an extended neutron distribution, and very few, if any, excited states.

This peculiar structure could affect the reaction properties. The loosely bound structure of halo nuclei should affect the collisions with heavy targets at energies around the Coulomb barrier. One can thus expect for the elastic process a departure from Rutherford scattering [1]. This deviation can shed light on the structure as well as on how the scattering process depends upon the coupling to the continuum.

\section{Scattering of halo nuclei on heavy target at Coulomb barrier energies}

\subsection{Experimental Set up @ ISAC-II TRIUMF}

The experiments were performed at the ISAC-II line at the TRIUMF facility (Vancouver, Canada). A primary proton beam of $500 \mathrm{MeV}$ energy and $100 \mu \mathrm{A}$ intensity irradiated a production target of

\footnotetext{
ae-mail: olof.tengblad@csic.es
}

This is an Open Access article distributed under the terms of the Creative Commons Attribution License 2.0, which permits unrestricted use, distribution, and reproduction in any medium, provided the original work is properly cited. 
Ta. The secondary beams of $\mathrm{Li}$ isotopes were transported to the ISAC-II facility for post-acceleration. The average intensity of the ${ }^{11} \mathrm{Li}$ beam, as detected in our monitor detector located $28 \mathrm{~cm}$ downstream of the ${ }^{208} \mathrm{~Pb}$ target, was $4.300{ }^{11} \mathrm{Li} / \mathrm{s}$.

The accelerated incoming Li-beam impinged on a $1.45 \mathrm{mg} / \mathrm{cm}^{2}{ }^{208} \mathrm{~Pb}$ target. The experimental setup consisted of four telescopes, T1-T4.Two of them, T1 and T2, were placed in the target forward direction, each one consisting of a $40 \mu \mathrm{m}$ thick double-sided silicon strip detector (DSSSD) [2] acting as $\Delta E$ detector and a $500 \mu \mathrm{m}$ thick Si-PAD as an $E$ detector. Telescopes T3 and T4 were placed in the backward direction, each having a $20 \mu \mathrm{m}$ thick $\Delta E$ single-sided silicon 16-strip detector and a 60 $\mu \mathrm{m}$ thick DSSSD behind. The segmentation of the detector system gives information from $2563 \times 3$ $\mathrm{mm}^{2}$ pixels per telescope. This configuration permitted high angular resolution $\left(2^{\circ}-3^{\circ}\right)$ with a large angular coverage from $10^{\circ}$ to $140^{\circ}$, see Fig. 1 .

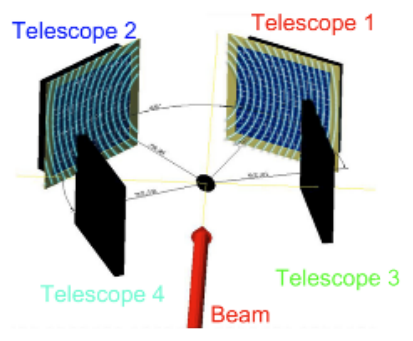

Figure 1. The figure shows the experimental set-up consisting of 4 particle telescopes T1 - T4 as described in the text.

\subsection{Reaction data from ${ }^{9} \mathrm{Li}$ and ${ }^{11} \mathrm{Li}$ beams on ${ }^{208} \mathrm{~Pb}$}

Due to the compact geometry used, a refined determination of the angle subtended by each pixel was done based on the fact that the elastic scattering of ${ }^{9} \mathrm{Li}$ on ${ }^{208} \mathrm{~Pb}$ at energies below the barrier follows the Rutherford scattering formula. Further details on the setup, data processing, and event selection can be found in Refs.[3,4]. Elastic events were selected in the two-dimensional plot of $\Delta E$ versus $\Delta E+E$ energy spectra for each pixel. A clear identification of the elastic peaks and fragments, both in the ${ }^{9} \mathrm{Li}$ and in the ${ }^{11} \mathrm{Li}$ scattering data, was achieved. Figure 2 illustrates the data obtained for ${ }^{9} \mathrm{Li}$ and ${ }^{11} \mathrm{Li}$ beams scattered on the same target and at equivalent c.m. energies. In the two-dimensional plot for the ${ }^{11} \mathrm{Li}$ scattered data, the contribution of the elastic channel and the ${ }^{9} \mathrm{Li}$ breakup data are clearly separated.
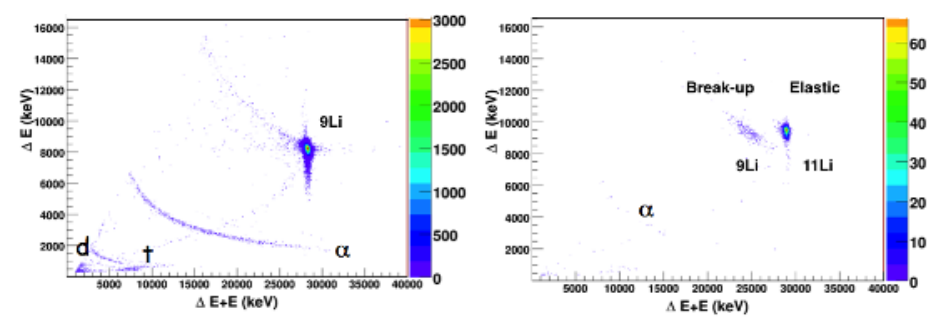

Figure 2. Experimental data obtained for ${ }^{9} \mathrm{Li}$ (left panel) and ${ }^{11} \mathrm{Li}$ (right panel) beams scattered on ${ }^{208} \mathrm{~Pb}$ target and at equivalent c.m. energies $(29 \mathrm{MeV})$. The data shown are taken in the same angular range $\left(24^{\circ}-26^{\circ}\right)$, and over the same time and so the huge difference in statistics can be noted. 


\subsection{First determination of Elastic Scattering of ${ }^{9} \mathrm{Li} \&{ }^{11} \mathrm{Li}$ around the Coulomb Barrier}

The scattering data of the core nucleus ${ }^{9} \mathrm{Li}$ follow the expected behavior for well-bound nuclei, obeying the Rutherford formula for energies below the Coulomb barrier and exhibiting a Fresnel-like diffraction pattern at energies around the coulomb barrier $(28 \mathrm{MeV})$. On the other hand, the ${ }^{11} \mathrm{Li}$ elastic cross section data departs significantly from the standard behavior of a well-bound nucleus, showing a strong reduction with respect to the Rutherford cross section both below and around the Coulomb barrier. This is in qualitative agreement with the prediction made in Ref. [1] obtained taking into account the effect of Coulomb dipole polarizability. In Fig. 3 is shown a three-body CDCC calculations using a 2-body structure of ${ }^{11} \mathrm{Li}$ and including Coulomb and nuclear couplings to all orders, that reproduces satisfactorily the experimental elastic angular distributions, for further details see Ref. [3] where also a more realistic four-body CDCC calculations is presented, taking into account the three-body nature of ${ }^{11} \mathrm{Li}$.
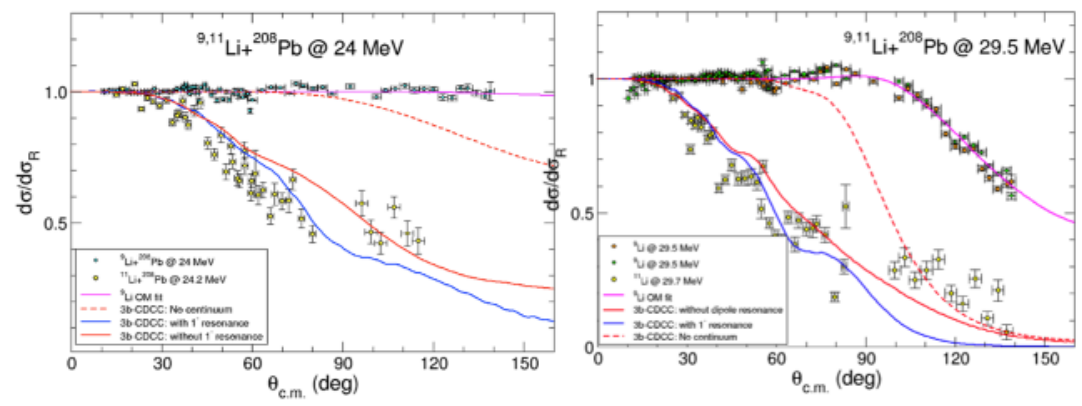

Figure 3. Elastic differential cross section of ${ }^{9} \mathrm{Li}$ and ${ }^{11} \mathrm{Li}{ }^{208} \mathrm{~Pb}$ at two different C.M. energies plotted as a ratio to the Rutherford cross section. For details see Ref. [3].

\subsection{Breakup probabilities of ${ }^{11} \mathrm{Li}$ on ${ }^{208} \mathrm{~Pb}$}

To quantify the importance of the break-up channel, we define a break-up probability as the ratio of ${ }^{9} \mathrm{Li}$ events to the sum of ${ }^{9} \mathrm{Li}$ and ${ }^{11} \mathrm{Li}$ events for each scattering angle. This magnitude is shown in Fig. 4 for the two incident energies. The errors indicated are purely statistical. In both cases, the breakup probability increases smoothly with increasing angle. We note that the large yield of ${ }^{9} \mathrm{Li}$ at backward angles is comparable to that of ${ }^{11} \mathrm{Li}$ at $24.3 \mathrm{MeV}$ and $70 \%$ larger at $29.8 \mathrm{MeV}$.

The experimental breakup probabilities have been compared with continuum-discretized coupledchannels (CDCC) calculations [5], using the four-body version of this method proposed in Ref.[6]. This is an extension of the standard CDCC method appropriate for three-body projectiles. In these calculations, the ${ }^{11} \mathrm{Li}$ states (bound and unbound) are described within a three-body model $\left({ }^{9} \mathrm{Li}+n+n\right)$. To reduce the complexity of the calculations, the spin of the ${ }^{9} \mathrm{Li}$ core is ignored. For the $n-{ }^{9} \mathrm{Li}$ and $n-n$ interactions we adopt the model P4 of Ref.[7].

\section{Conclusion}

Elastic and break-up cross section data for ${ }^{9} \mathrm{Li} \&{ }^{11} \mathrm{Li}$ on ${ }^{208} \mathrm{~Pb}$ at energies near the Coulomb barrier were obtained for first time. The experimental system is able to separate the elastic ejectiles and fragments even at low energy and statistics. The ${ }^{11} \mathrm{Li}$ elastic cross section depart strongly from Rutherford 

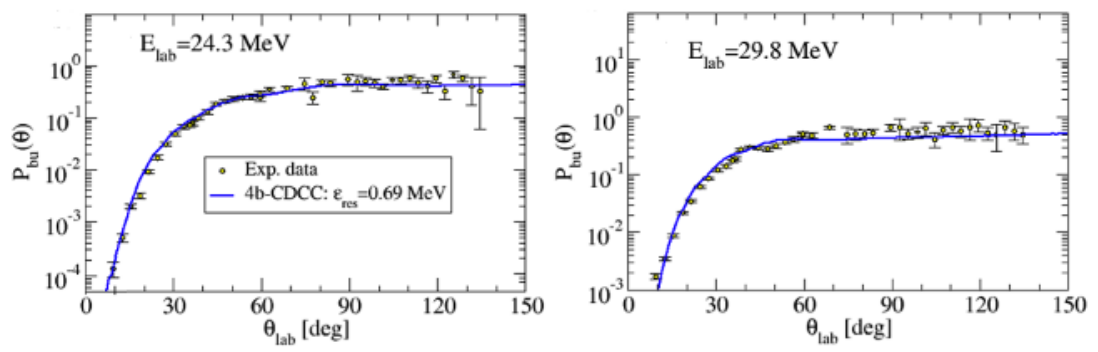

Figure 4. The angular distribution of the Breakup probabilities of ${ }^{11} \mathrm{Li}$ on ${ }^{208} \mathrm{~Pb}$ at $24.3 \mathrm{MeV}$ and $29.8 \mathrm{MeV}$. The circles represent the experimental data. For details see Ref. [4].

behavior at energies well below the barrier. The behavior is well described by CDCC calculations when Coulomb and continuum couplings are taken into account. Break-up cross sections are very large, but are also well predicted by the CDCC calculations.

In order to better understand the physics involved we have performed a second experiment, this time on the one-neutron halo ${ }^{11} \mathrm{Be}$ (experiment S1202 at TRIUMF). From theoretical point of view this should be an easier case but from the point of experiment more difficult as also the inelastic channel via the $320 \mathrm{KeV}$ excited state of ${ }^{11} \mathrm{Be}$ is available. For this reason the experimental set-up was changed to include the TIGRESS [8] gamma ball at TRIUMF. Data were taken using beams of ${ }^{10} \mathrm{Be}$ and ${ }^{11} \mathrm{Be}$ during 2012 on a ${ }^{208} \mathrm{~Pb}$ target and in 2013 on a ${ }^{197} \mathrm{Au}$ target. The raw data show very good separation of the three reaction channels. These data are still under analysis and will be published elsewhere.

\section{Acknowledgements}

This work was supported by the Spanish CICYT under the project numbers FPA2009-07387, FPA2012-32443, FPA2009-08848, FPA2009-07653, and FPA2010-22131-C02-01 and by the Consolider Ingenio 2010 Program CPAN (CSD2007- 00042). We also acknowledge all support during the experiments from the TRIUMF staff.

\section{References}

[1] M.V. Andres and J. Gomez-Camacho, Phys. Rev. Lett. 82, 1387 (1999).

[2] O. Tengblad, et.al., Nucl. Instrum. Methods Phys. Res., A525, 458 (2004).

[3] M. Cubero, et.al., Phys. Rev. Lett. 109, 262701 (2012)

[4] J.P. Fernandez-Garcia, et.al., Phys. Rev. Lett. 110, 142701 (2013)

[5] N. Austern, et.al., Phys. Rep. 154, 125 (1987).

[6] M. Rodriguez-Gallardo, et.al., Phys. Rev. C 80, 051601(R) (2009).

[7] I. J. Thompson and M. V. Zhukov, Phys. Rev. C 49, 1904 (1994).

[8] M.A. Schumaker, et.al., Nucl. Instrum. Methods Phys. Res., A570, 437 (2007). 